\title{
Oxygen, oxidative stress, hypoxia, and heart failure
}

\author{
Frank J. Giordano
}

Department of Medicine, Yale University School of Medicine, New Haven, Connecticut, USA.

\begin{abstract}
A constant supply of oxygen is indispensable for cardiac viability and function. However, the role of oxygen and oxygen-associated processes in the heart is complex, and they and can be either beneficial or contribute to cardiac dysfunction and death. As oxygen is a major determinant of cardiac gene expression, and a critical participant in the formation of ROS and numerous other cellular processes, consideration of its role in the heart is essential in understanding the pathogenesis of cardiac dysfunction.
\end{abstract}

The mammalian heart is an obligate aerobic organ. At a resting pulse rate, the heart consumes approximately $8-15 \mathrm{ml}$ $\mathrm{O}_{2} / \mathrm{min} / 100 \mathrm{~g}$ tissue. This is significantly more than that consumed by the brain (approximately $3 \mathrm{ml} \mathrm{O} / \mathrm{min} / 100 \mathrm{~g}$ tissue) and can increase to more than $70 \mathrm{ml} \mathrm{O}_{2} / \mathrm{min} / 100$ g myocardial tissue during vigorous exercise $(1,2)$. Mammalian heart muscle cannot produce enough energy under anaerobic conditions to maintain essential cellular processes; thus, a constant supply of oxygen is indispensable to sustain cardiac function and viability. The story of oxygen in the heart is complex, however, and goes well beyond its role in energy metabolism.

Oxygen is a major determinant of myocardial gene expression, and as myocardial $\mathrm{O}_{2}$ levels decrease, either during isolated hypoxia or ischemia-associated hypoxia, gene expression patterns in the heart are significantly altered (3). Oxygen participates in the generation of $\mathrm{NO}$, which plays a critical role in determining vascular tone, cardiac contractility, and a variety of additional parameters. Oxygen is also central in the generation of reactive oxygen species (ROS), which can participate as benevolent molecules in cell signaling processes or can induce irreversible cellular damage and death. Oxygen is thus both vital and deleterious (4).

\section{The role of oxygen in myocardial energetics and metabolism}

The heart can utilize a variety of metabolic fuels, including fatty acids, glucose, lactate, ketones, and amino acids. In the fed state, fatty acids are the preferred fuel, accounting for up to $90 \%$ of the total acetyl-CoA provided to cardiac mitochondria (5). Fatty acids are metabolized by $\beta$-oxidation, producing acetyl-CoA, NADH, and $\mathrm{FADH}_{2}$. The acetyl-CoA enters the Krebs cycle, producing more $\mathrm{NADH}$ and $\mathrm{FADH}_{2}$. Glucose is metabolized initially via the glycolytic pathway, producing a relatively small amount of ATP and also pyruvate, which enters the Krebs cycle, producing NADH and $\mathrm{FADH}_{2}$. In the absence of oxygen, the total amount of energy produced by these processes is insufficient to meet cardiac needs. The cardiac energy requirement is met, however, by entry of the resultant $\mathrm{NADH}$ and $\mathrm{FADH}_{2}$ into the electron transport chain, which gener-

Nonstandard abbreviations used: ARNT, aryl hydrocarbon nuclear translocase; ASK-1, apoptosis-signaling kinase 1; ATII, angiotensin II; HIF-1 $\alpha$, hypoxia-inducible factor $1 \alpha$; MI, myocardial infarction; NOS, NO synthase; SOD, superoxide dismutase; VHL, von Hippel-Lindau protein; $\mathrm{XO}$, xanthine oxidase.

Conflict of interest: The author has declared that no conflict of interest exists.

Citation for this article: $J$. Clin. Invest. 115:500-508 (2005).

doi:10.1172/JCI200524408 ates ATP by oxidative phosphorylation in the mitochondria. Oxygen serves as the terminal electron acceptor in the electron transport chain, and in the absence of sufficient oxygen, electron transport ceases and cardiac energy demands are not met (Figure 1).

\section{Generation and counterbalancing of ROS}

ROS can be formed in the heart by a variety of mechanisms, including generation during oxidative phosphorylation in the mitochondria as a byproduct of normal cellular aerobic metabolism $(4,6)$. Thus, the major process from which the heart derives sufficient energy can also result in the production of ROS (6). Each oxygen atom contains 2 unpaired electrons in its outermost shell. Atoms or molecules with unpaired electrons are designated free radicals and are highly reactive entities that can readily participate in a variety of chemical/biochemical reactions. Molecular oxygen, $\mathrm{O}_{2}$, is characterized as diradical, a property that allows liquid oxygen to be attracted to the poles of a magnet. This property also dictates that full reduction of oxygen to water as a terminal event in the electron transport chain requires 4 electrons. The sequential donation of electrons to oxygen during this process can generate ROS as intermediates, and "electron leakage" can also contribute to the formation of $\operatorname{ROS}(4,7,8)$. Donation of a single electron to molecular oxygen results in the formation of the superoxide radical $\left(\mathrm{O}_{2}{ }^{--}\right)$. Donation of a second electron yields peroxide, which then undergoes protonation to yield hydrogen peroxide $\left(\mathrm{H}_{2} \mathrm{O}_{2}\right)$. Donation of a third electron, such as occurs in the Fenton reaction $\left(\mathrm{Fe}^{2+}+\mathrm{H}_{2} \mathrm{O}_{2}\right.$ $\left.\rightarrow \mathrm{Fe}^{3+}+{ }^{\cdot} \mathrm{OH}+\mathrm{OH}^{-}\right)$, results in production of the highly reactive hydroxyl radical $(\cdot \mathrm{OH})$. Finally, donation of a fourth electron yields water. Singlet oxygen $\left({ }^{1} \mathrm{O}_{2}\right)$, a very short-lived and reactive form of molecular oxygen in which the outer electrons are raised to a higher energy state, can be formed by a variety of mechanisms, including the Haber-Weiss reaction $\left(\mathrm{H}_{2} \mathrm{O}_{2}+\mathrm{O}_{2}{ }^{--} \rightarrow \cdot \mathrm{OH}+\mathrm{OH}^{-}+{ }^{1} \mathrm{O}_{2}\right)$ (9).

ROS can be formed in the heart, and other tissues, by several mechanisms; they can be produced by xanthine oxidase $(\mathrm{XO})$, $\mathrm{NAD}(\mathrm{P}) \mathrm{H}$ oxidases, cytochrome $\mathrm{P} 450$; by autooxidation of catecholamines; and by uncoupling of NO synthase (NOS) (10-14). $\mathrm{NO}$ contains an unpaired electron, and under certain conditions can react with $\mathrm{O}_{2}^{--}$to form peroxynitrite $\left(\mathrm{ONOO}^{--}\right)$, a powerful oxidant. ROS formation in the heart can be induced by the action of cytokines and growth factors as well. Angiotensin II (ATII), PDGF, and TNF- $\alpha$, for example, can induce $\mathrm{H}_{2} \mathrm{O}_{2}$ and $\mathrm{O}_{2}{ }^{--}$formation via activation of the NAD $(\mathrm{P}) \mathrm{H}$ oxidases $(10,15)$. This $\mathrm{NAD}(\mathrm{P}) \mathrm{H}$ dependent pathway is best described in vascular smooth muscle cells but has also been documented in other cell types, including 
A

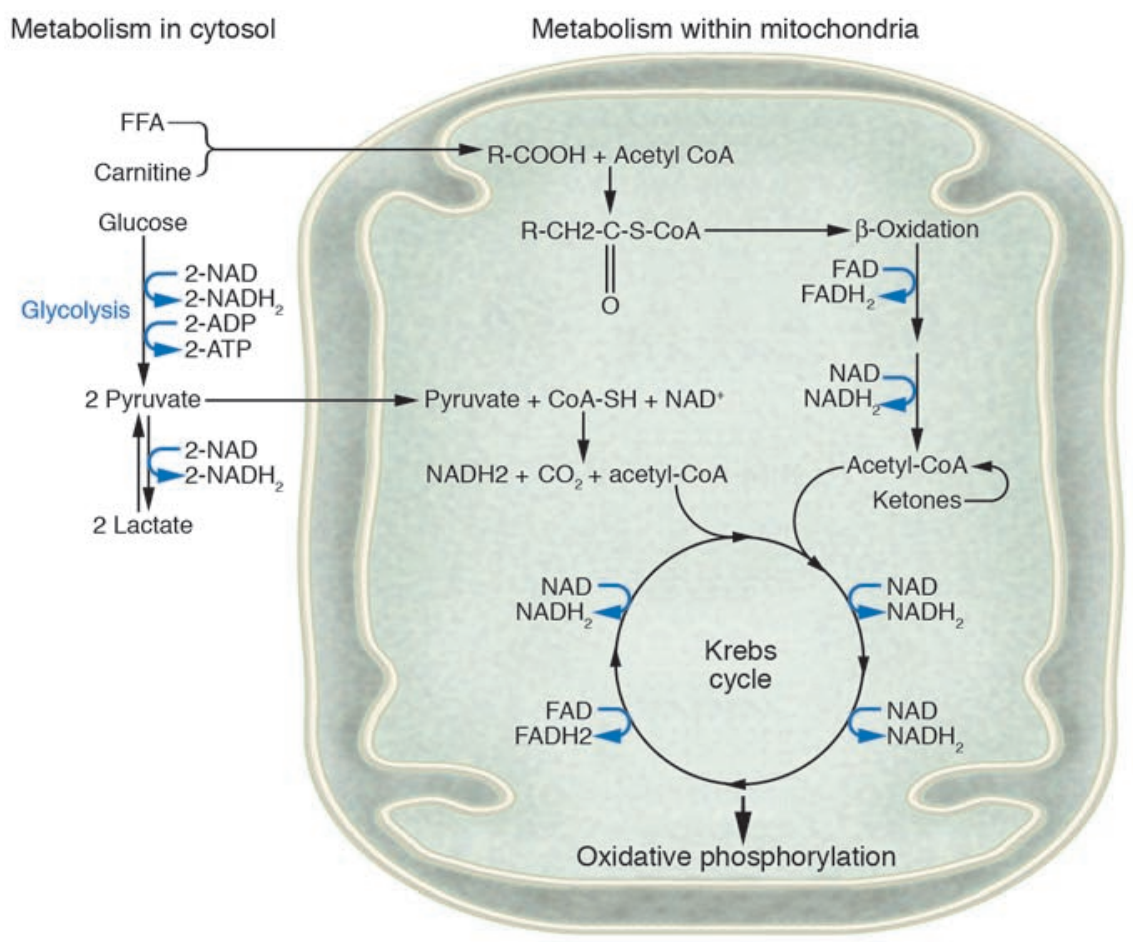

B

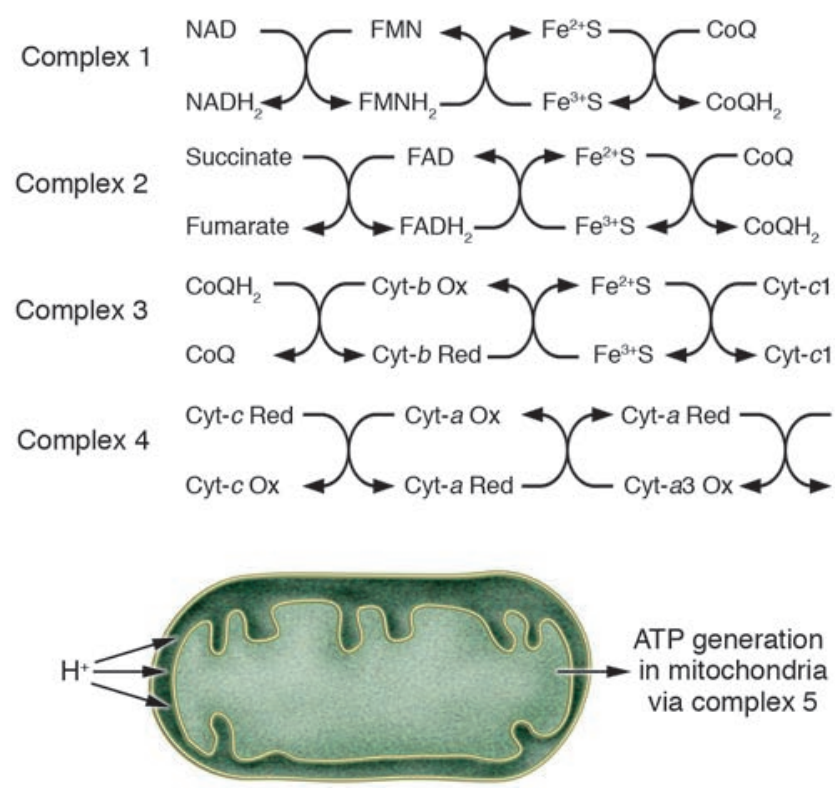

Figure 1

Role of oxygen in myocardial metabolism. (A) Schematic depiction of the pathways by which cardiac muscle utilizes various fuels, including fatty acids, glucose, lactate, and ketones. Glycolysis occurs in the cytosol and does not require oxygen. $\beta$-Oxidation of fatty acids, ketone metabolism, and the metabolism of glucose-derived intermediates all generate reduced flavoproteins $\left(\mathrm{NADH}_{2}\right.$ and $\mathrm{FADH} \mathrm{H}_{2}$ ). (B) Schematic depiction of the process of oxidative phosphorylation in the mitochondria. Complexes 1-4 refer to specific electron transfer steps that occur in the mitochondria. A series of electron transfers among the flavoproteins $\left(\mathrm{FMNH}_{2}\right.$, $\mathrm{NADH}_{2}, \mathrm{FADH}_{2}$ ), iron-sulfur, coenzyme $\mathrm{Q}$, and the cytochromes a-c1, results in accumulation of protons in the space between the inner and outer mitochondrial membranes. This proton gradient provides the energy for ATP production via complex 5 . Sustaining this crucial process requires the continuous availability of oxygen as the terminal electron acceptor in the chain. $\mathrm{Fe}^{2+\mathrm{S}}$, reduced iron-sulfur; $\mathrm{Fe}^{3+\mathrm{S}}$, oxidized iron-sulfur; FMN, flavin mononucleotide; cyt, cytochrome; CoQ, coenzyme Q.

antioxidant and redox regulatory system that has been implicated in a wide variety of ROS-related processes (21). Thioredoxin and thioredoxin reductase can catalyze the regeneration of many antioxidant molecules, including ubiquinone (Q10), lipoic acid, and ascorbic acid, and as such constitute an important antioxidant defense against ROS. Deletion of thioredoxin reductase results in developmental heart abnormalities and in cardiac death secondary to a severe dilated cardiomyopathy (26).

Nonenzymatic mechanisms include intracellular antioxidants such as the vitamins $\mathrm{E}, \mathrm{C}$, and $\beta$-carotene (a precursor to vitamin A), ubiquinone, lipoic acid, and urate (21). They also include glutathione, which acts as a reducing substrate for the enzymatic activity of glutathione peroxidase.

\section{The biological significance of ROS}

ROS have an important role in several important biological processes, including

cardiomyocytes (16-20). A number of additional ligands have been associated with the induction of ROS, including several with particular relevance to the cardiovascular system (reviewed by Thannickal et al. in ref. 15).

There are several cellular mechanisms that counterbalance the production of ROS, including enzymatic and nonenzymatic pathways (21). Among the best-characterized enzymatic pathways are catalase and glutathione peroxidase, which coordinate the catalysis of $\mathrm{H}_{2} \mathrm{O}_{2}$ to water, and the superoxide dismutases (SODs), which facilitate the formation of $\mathrm{H}_{2} \mathrm{O}_{2}$ from $\mathrm{O}_{2}{ }^{--}$(22-25). Thioredoxin and thioredoxin reductase together form an additional enzymatic the oxidative burst reaction essential to phagocytes (27). They are involved in a variety of cellular signaling pathways (28), acting in some instances as second messengers downstream of specific ligands, including TGF- $\beta 1$, PDGF, ATII, FGF-2, endothelin, and others $(14,15,29,30)$. ROS are also involved in modulating the activity of specific transcription factors, including NF- $\kappa$ B and activator protein-1 (AP-1) (20,31-34). NF- $\mathrm{B}$, for example, becomes more transcriptionally active in response to the contribution of ROS to the degradation of IKB, the inhibitory partner of NF- $\mathrm{KB}$ that sequesters it in the cytosol. Thus ROS can play an important role in modulating inflammation. 


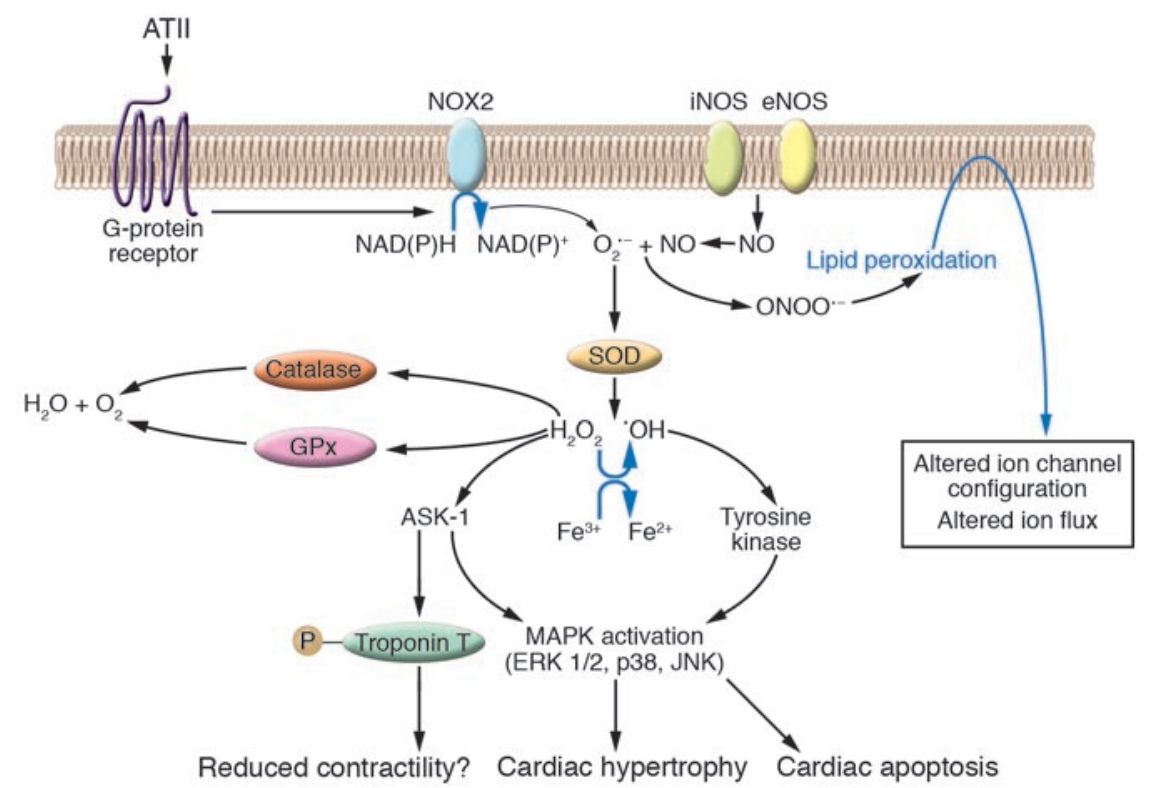

\section{Figure 2}

Mechanisms by which ROS can alter the structure and function of cardiac muscle. ATII binds a G-protein-associated receptor, initiating a cascade of events that involves activation of $\mathrm{O}_{2}{ }^{--}$production by the $\mathrm{NAD}(\mathrm{P}) \mathrm{H}$ oxidase NOX2. $\mathrm{O}_{2}^{--}$is converted by SOD into $\mathrm{H}_{2} \mathrm{O}_{2}$ and $\cdot \mathrm{OH}$ that mediates activation of MAPKs via a tyrosine kinase. MAPK activation can lead to cardiac hypertrophy or to apoptosis. The ROS that is generated can also signal through ASK1 to induce cardiac hypertrophy, apoptosis, or phosphorylate troponin $\mathrm{T}$, an event that reduces myofilament sensitivity and cardiac contractility. NO production by the NO synthases iNOS and eNOS can interact with $\mathrm{O}_{2}{ }^{--}$to form ONOO-- . ONOO-- can cause lipid peroxidation, an event that can alter ion channel and ion pump function. Catalase and glutathione reductase (GPx) are shown as enzymatic pathways to produce water and oxygen from $\mathrm{H}_{2} \mathrm{O}_{2}$.
Perhaps the most widely recognized biological effects of ROS, however, are those that occur when cellular antioxidant defenses are overwhelmed and ROS react directly with cellular lipids, proteins, and DNA, causing cell damage and death $(4,27,35,36)$. Lipid peroxidation, for example, is a well-characterized effect of ROS that results in damage to the cell membrane as well as to the membranes of cellular organelles $(37,38)$. ROS can contribute to mutagenesis of DNA by inducing strand breaks, purine oxidation, and protein-DNA cross-linking, and other ROSmediated alterations in chromatin structure may significantly affect gene expression $(39,40)$. Modification of proteins by ROS can cause inactivation of critical enzymes and can induce denaturation that renders proteins nonfunctional (41, 42). General aging and age-related alteration in the cardiovascular system have been attributed to the long-term cumulative effects of ROS, although the relative contribution of ROS to the aging process remains the subject of debate $(43,44)$. Figure 2 depicts several pathways by which ROS can mediate biological effects germane to the cardiovascular system.

\section{Actions of ROS relevant to the heart, and the potential role of ROS in heart failure}

A significant number of in vitro and animal studies have demonstrated ROS activation in the cardiovascular system in response to various stressors and in the failing heart $(6,14,20$, $36,45-47)$. Further, although the results are somewhat inconsistent, animal studies have also delineated that antioxidants and ROS defense pathways can ameliorate ROS-mediated cardiac abnormalities (26, 48-50).

Many ROS-mediated biological processes that are germane to the heart and to the genesis of heart failure have been described.

\section{ROS in ischemic syndromes}

Given that coronary artery disease (CAD) with consequent myocardial ischemia and necrosis is a leading cause of heart failure worldwide, it is important to note that ROS may play an important role in the genesis and progression of $\operatorname{CAD}(51,52)$. ROS activity in the vessel wall, for example, is thought to contribute to the forma- tion of oxidized LDL, a major contributor to the pathogenesis of atherosclerosis (53). ROS-associated activation of MMPs may play an important role in vessel plaque rupture, initiating coronary thrombosis and occlusion (54).

In the setting of acute myocardial infarction (MI), ROS are purported to play a significant role in tissue necrosis and reperfusion injury $(55,56)$. Transgenic overexpression of SOD has been shown to reduce infarct size in mice, which supports the contention that ROS are important mediators of myocardial damage in MI (50). However, attempts to target SOD to improve outcome after MI in other animal models have yielded mixed results, suggesting that ROS activation is only a single contributor to post-MI necrosis and reperfusion injury and/or that SOD alone is insufficient to neutralize the deleterious effects of ROS in this setting. ROS also play a significant role in the pathogenesis of myocardial stunning, which can complicate acute ischemic syndromes (57). XO expression is significantly increased in the setting of MI, which increases the probability that XO-induced ROS will be generated. Interestingly, administration of the $\mathrm{XO}$ inhibitor allopurinol in the setting of acute MI attenuates stunning and ameliorates the excitation-contraction uncoupling that occurs in stunned myocardium $(58,59)$.

At least $20 \%$ of patients who suffer MI go on to develop heart failure. The manner in which the ventricle heals and remodels after MI is a major determinant of eventual cardiac function and the progression to heart failure (60). ROS may contribute to the remodeling processes in a number of ways, including activating MMPs that participate in reconfiguration of the extracellular matrix; acting as signaling molecules in the development of compensatory hypertrophy; and contributing to myocyte loss via apoptosis or other cell death mechanisms $(20,61,62)$. Recently it was shown that inhibition of XO with allopurinol after experimental $\mathrm{MI}$ in dogs diminished ROS production in the myocardium and attenuated maladaptive $L V$ remodeling, leading to improved postMI cardiac function (63). While this does not prove that ROS are a major clinical target for decreasing the progression to failure after MI, these findings are part of a growing list of data suggesting a major contribution of ROS to this process. 


\section{ROS in cardiac hypertrophy, apoptosis, and the transition to failure}

Cardiac hypertrophy can be either compensatory and adaptive or a maladaptive precursor to cardiac failure. Mounting evidence has strongly implicated ROS signaling in the genesis of cardiac hypertrophy (64-68). Many extracellular factors are capable of inducing hypertrophy of cardiomyocytes, and many of the various downstream signaling pathways that mediate the hypertrophic growth response to these factors can be activated directly or indirectly by ROS. These include PKC; the MAPKs p38, JNK, apoptosis-signaling kinase 1 (ASK-1), and ERK1/2; PI3K; Akt; several tyrosine kinases (e.g., src and FAK); NF-кB; and calcineurin (20, 69). An example of purported direct activation is ROS-mediated activation of PKC via oxidation of cysteine residues (70). An example in which an extracellular signal induces cardiac hypertrophy via a ROS-dependent pathway is ATII-induced hypertrophy. The clinical role of ATII in the development and progression of heart failure is now well established, and blocking either ATII generation or ATII binding to the AT1 receptor prolongs life in specific patient cohorts. ATII induces cardiac hypertrophy via a G-protein-linked pathway that involves generation of ROS and ROS-associated activation of several downstream signals, including MAPKs (71). Interestingly, inhibition of ROS by antioxidants blocks ATII-mediated cardiac hypertrophy $(71,72)$.

As mentioned above, it has been shown that ATII induces ROS in large measure via $\mathrm{NAD}(\mathrm{P}) \mathrm{H}$ oxidases, although the mechanism by which ATII activates these oxidases has remained incompletely understood. In vitro and in vivo data suggest that the small G-protein Rho may be involved in this link. Inhibition of Rho with a dominant-negative construct in neonatal rat cardiomyocytes prevents ATII-mediated intracellular oxidation events, and inhibition of Rho activation by the HMG-CoA reductase inhibitor simvastatin blocked ATII-mediated increases in protein synthesis in these cells, leading to smaller cell sizes $(20,73,74)$. Simvastatin administration in vivo also prevented cardiac hypertrophy in response to either ATII administration or pressure overload induced by aortic banding, which establishes that the effects of ROS on hypertrophy are not an artifactual in vitro finding (74).

Another mechanism by which ROS can induce cardiac hypertrophy is via transcription factor-mediated alterations in gene expression. For example, ATII stimulates ROS-mediated activation of the transcription factor NF- $\mathrm{BB}$, which is purported to play an important role in the induction of cardiac hypertrophy. AP-1 activity is also purportedly regulated by ROS and is involved in the transcriptional expression of several genes involved in cardiac hypertrophy. In addition, it is possible that regulation of transcription by ROS may be far more widespread than anticipated and that ROS-mediated alterations in gene structure and function contribute significantly to the pathogenesis of disease in many organ systems, including the heart. Recently the roles of ROS in chromatin remodeling and in DNA damage have become more established, and consideration of the potential involvement of these effects in the failing heart is warranted.

Another MAPK family member linking ROS and hypertrophy is the redox-sensitive kinase ASK-1. ASK-1 is strongly activated by ROS and in turn activates MAPKs p38 and JNK. Deletion of ASK-1 attenuates p38 and JNK activation and the cardiac hypertrophic response to ATII (75). Expression of a dominant-negative ASK-1 attenuates NF- $\mathrm{KB}$ activation and inhibits cardiac hypertrophy in response to ROS-generating G-protein receptor agonists, which demonstrates a role for ASK-1 in the link among ROS, NF- $\kappa$ B, and cardiac hypertrophy (31). ASK- 1 also mediates TNF- $\alpha$-induced apoptosis, which constitutes another link between ROS and an event (apoptosis) that contributes to the pathogenesis of heart disease (76). Overexpression of ASK-1 induces apoptosis in cardiomyocytes, and ASK-1-null mice demonstrate attenuated ventricular remodeling in response to pressure overload, a finding attributed in part to a reduction in cardiac apoptosis (77).

Cardiomyocyte apoptosis occurs in hypertrophied, ischemic, and failing hearts and may contribute to the development and progression of cardiac dysfunction and heart failure $(47,78)$. Experimental evidence suggests that ROS can mediate apoptosis by a variety of mechanisms, including direct mediation of genotoxicity $(62,68,79)$. Interestingly, whether or not apoptosis is induced in cardiomyocytes by oxidative stress appears to be dependent upon the level of ROS produced (64). For instance, in adult cardiomyocytes, relatively low levels of $\mathrm{H}_{2} \mathrm{O}_{2}$ are associated with the activation of ERK1/2 MAPK and the stimulation of protein synthesis. Conversely, a higher level of $\mathrm{H}_{2} \mathrm{O}_{2}$, while still activating ERK1/2, also activates the JNK and p38 MAPKs and Akt and induces apoptosis (64). Another clinically relevant potential link between heart failure and ROS involves adrenergic signaling (14, $80)$. That $\beta$-adrenergic receptor ( $\beta$ AR) blockers prolong life and are beneficial in heart failure is well established. One mechanism may be the prevention of $\beta A R$-induced apoptosis, an event that appears to involve ROS activation (80).

\section{ROS effects on ion channels and calcium flux}

Ion flux is critical to normal cardiac function, and there is significant evidence that ROS alter ion channel flux and membrane ion pump function in a biologically important manner in heart muscle (81). General membrane damage secondary to ROS-mediated lipid peroxidation is one mechanism by which this can occur; however, more specific ROS-mediated effects also contribute. ROS can target L-type calcium channels on the sarcolemma and suppress the $\mathrm{Ca}^{2+}$ current (82). ROS depress the activity of the sarcoplasmic reticulum $\mathrm{Ca}^{2+}$ ATPase SERCA2, a membrane calcium pump that has been shown to play a crucial role in cardiac calcium handling and as a determinant of myocardial contractility (83). SERCA2 expression is concomitantly reduced in cardiomyocytes stimulated to hypertrophy via ROS-associated signaling pathways. ROS generation can also alter the function of cardiac sodium channels, potassium channels, and ion exchangers, such as the $\mathrm{Na} / \mathrm{Ca}$ exchanger $(84,85)$.

In another ROS-mediated pathway that may lead to reduced contractility, ROS can decrease the calcium sensitivity of the myofilaments. Recently it was shown that the ROS-related kinase ASK-1 associates with and phosphorylates troponin $\mathrm{T}$ in vitro and in vivo and that this event diminishes contractility and alters calcium handling in cardiomyocytes (86). Whether this pathway contributes to human heart failure remains unknown. It has been postulated, however, that via mechanisms such as this ROS-mediated abnormality in excitation-contraction coupling, chronic exposure to ROS contributes to the progression of failure.

\section{Lipotoxicity and ROS}

Myocardial lipotoxicity refers to the accumulation of intramyocardial lipids concomitant with contractile dysfunction, often associated with myocyte death $(87,88)$. Recently it was shown that lipid accumulation is a significant feature of clinical heart failure (88). Lipid accumulation occurs when there is an imbalance between 
lipid uptake and $\beta$-oxidation, a circumstance that can occur via a variety of mechanisms. Lipid accumulation induces an increase in the PPAR $\alpha$, a nuclear receptor that alters gene expression in response to lipids. PPAR $\alpha$ increases fatty acid oxidation, and increased expression of PPAR $\alpha$ has been associated with the development of cardiac dysfunction, including diabetic cardiomyopathy (89). Although the mechanism by which this occurs remains unclear, $\beta$-oxidation of fatty acids generates ROS, and data suggests that ROS play a role in the pathogenesis of PPAR $\alpha$-associated cardiomyopathies and lipotoxicity (88).

\section{Reactive nitrogen species, NO, and cardiac function}

$\mathrm{NO}$, by virtue of its unpaired outer shell electron, is a reactive molecule. In addition to the expansive array of crucially important biological processes in which it plays a role, $\mathrm{NO}$ is also a determinant of cardiac contractility. Initially NO was characterized as a contractility depressant, although the role of $\mathrm{NO}$, and the NO-generating NO synthases, is now understood to be much more complex with regard to effects on cardiac contractility $(90,91)$. Although its general biology will not be reviewed here, NO does react and interact with ROS, and this crosstalk can also have significant effects on cardiac function (92).

NO can mediate the $S$-nitrosylation of proteins at specific cysteine residues (93). This process also occurs in the heart and has significant functional implications, especially with regard to calcium flux and excitation-contraction coupling $(94,95)$. $S$-nitrosylation is facilitated by $\mathrm{O}_{2}{ }^{--}$when $\mathrm{O}_{2}{ }^{--}$is present at "physiologic" levels. When levels of $\mathrm{O}_{2}{ }^{--}$increase, however, it becomes inhibitory to normal $\mathrm{S}$-nitrosylation. Increased $\mathrm{O}_{2}{ }^{--}$levels also facilitate interaction of $\mathrm{O}_{2} \cdot-$ with $\mathrm{NO}$ to form deleterious reactive molecules, including peroxynitrite (96). Thus, at an optimal $\mathrm{NO} / \mathrm{O}_{2}{ }^{--}$stoichiometry, the crosstalk between these 2 reactive species facilitates essential cellular processes, a relationship termed nitroso-redox balance in a recent editorial addressing the results of the African American Heart Failure Trial (95). In that trial, combined therapy with hydralazine, a vasodilator that inhibits generation of $\mathrm{O}_{2}^{--}$, and isosorbide dinitrate improved quality-oflife scores and decreased mortality by approximately $45 \%$ in African Americans with severe heart failure (97). A compelling argument has been made that the effectiveness of this therapy is due in part to restoration of nitroso-redox balance.

\section{Do ROS play a role in clinical heart failure?}

Several clinical observations support the hypothesis that ROS play a role in human heart failure, although to date the clinical data has been conflicting and less than compelling $(98,99)$. In a handful of defined cardiomyopathies, the contribution of ROS is well established, including alcohol-mediated and anthrocyclineinduced cardiomyopathies (100). In other forms of human heart failure, the role of ROS has not been definitively established. One problem is that it has been difficult to determine ROS activity in vivo, and clinical studies have relied on indirect measures, using biochemical markers of ROS activity, including indices of lipid peroxidation. Further, the patients included in these studies often had confounding comorbidities that could induce alterations in these biochemical markers, independent of cardiac-specific ROS activity (99). Finally, the presence of ROS in the heart concomitant with heart failure does not prove a causal relationship, and clinical trials using antioxidant therapy have yielded mixed and often disappointing results. This may, however, reflect the difficulty in altering ROS-associated processes in vivo rather than an absence of their involvement in human cardiac failure or may indicate that the relationship between ROS and heart failure is too complex to be addressable by a single intervention (101). Representative examples of the conflicting clinical data follow.

In failing human hearts of patients with either ischemic or dilated cardiomyopathy, $\mathrm{NAD}(\mathrm{P}) \mathrm{H}$ oxidase-linked ROS activity was elevated and associated with increased rac-1 GTPase activity (98). Treatment of heart failure patients with statins decreased rac- 1 activity in myocardium from these patients, possibly via statin effects on ROS activity. Treatment with the XO inhibitor allopurinol improved cardiac contractility and restored normal vasomotor reactivity $(102,103)$. Treatment with vitamin $C$ inhibited endothelial cell apoptosis (104). Carvedilol has been shown in clinical trials to be superior to other $\beta$-blockers for the treatment of congestive heart failure, and one potential mechanism that has been put forth to explain this is that carvedilol has antioxidant effects (105). Conversely, large clinical trials of the antioxidant vitamins or precursors have not shown benefit in preventing cardiac morbidity or mortality (106-108). A larger clinical trial of a $\mathrm{XO}$ inhibitor to follow up on the earlier positive clinical results is planned, but at this time, the jury remains out regarding the therapeutic utility of antioxidant therapy for heart failure (109).

\section{Oxygen as a determinant of myocardial gene expression and cardiac function}

Myocardial oxygen consumption and availability must be matched to ensure normal cardiac function and viability. Although physiological mechanisms are in place to coordinate this balance dynamically in response to acute and subacute alterations in cardiac workload, alterations in gene expression patterns also play a critical role, generally as adaptive responses to cardiac stressors that alter either myocardial $\mathrm{O}_{2}$ consumption (e.g., pressure overload) or $\mathrm{O}_{2}$ delivery (e.g., coronary artery disease). Gene expression is adjusted to oxygen availability in the heart by several mechanisms, including regulation of gene transcription by the hypoxia-inducible basic helix-loop-helix transcription factor hypoxia-inducible factor $1 \alpha$ $($ HIF-1 $\alpha)(3,110)$. HIF- $1 \alpha$ regulates the transcription of an extensive repertoire of genes, including many involved in angiogenesis and vascular remodeling, erythropoiesis, metabolism, apoptosis, control of ROS, vasomotor reactivity and vascular tone, and inflammation (111-113).

HIF- $1 \alpha$ alters the transcription of these genes by dimerizing with the aryl hydrocarbon nuclear translocase (ARNT, or HIF-1 $\beta$ ) and then binding to specific hypoxia response elements (HREs) in their regulatory regions. The general response to HIF is transcriptional activation, coordinated by recruitment of the p300 coactivator. ARNT is generally abundant; thus the availability of HIF- $1 \alpha$ is rate limiting in formation of the HIF-ARNT dimer. The major mechanism coordinating the effects of HIF- $1 \alpha$ on gene expression with oxygen availability involves the posttranslational regulation of HIF- $1 \alpha$ abundance. HIF- $1 \alpha$ is constitutively transcribed and translated in the heart and most other tissues, but under conditions in which oxygen is abundant, HIF undergoes prolyl-hydroxylation at the direction of specific cellular prolyl hydroxylases (114). This hydroxylation event produces a binding site recognized by the von Hippel-Lindau protein (VHL) (115). VHL, which is deficient in the von Hippel-Lindau syndrome, is a subunit in an E3 ubiquitin ligase complex that polyubiquitylates HIF- $1 \alpha$, thus targeting it for rapid destruction by the proteosome. When oxygen is less abun- 


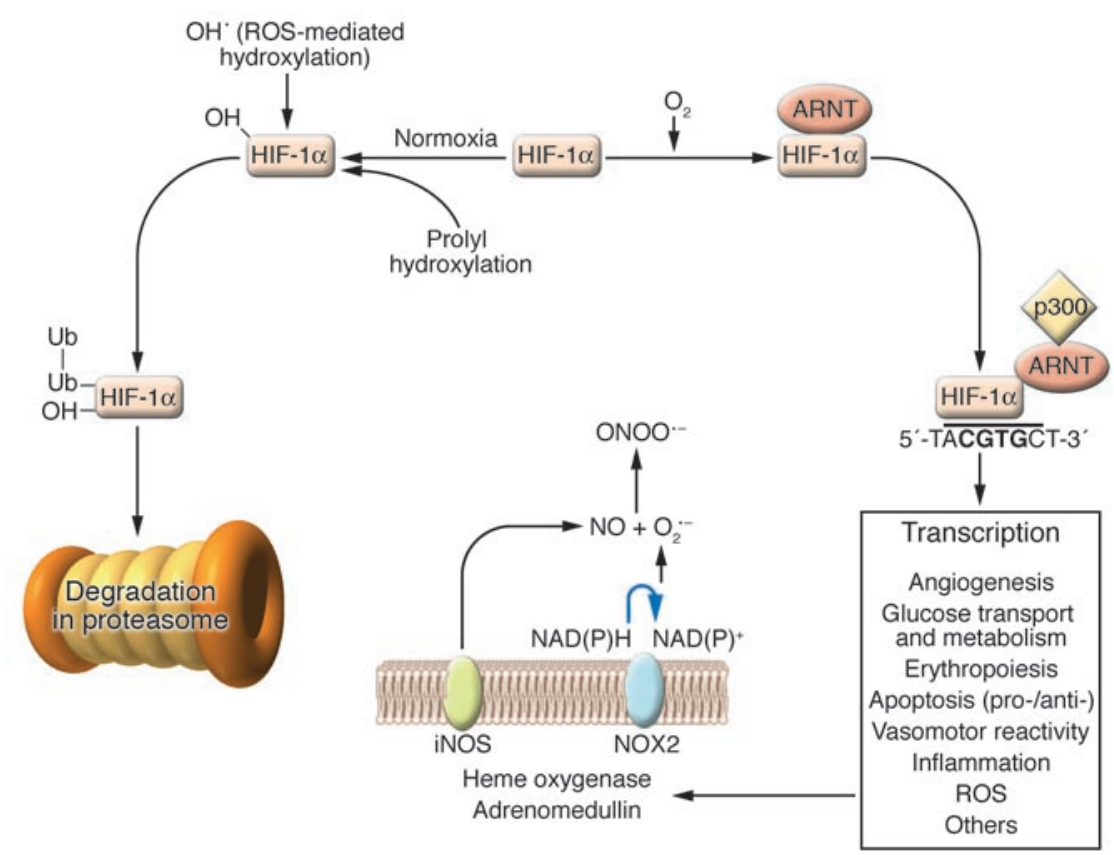

\section{Figure 3}

Transcriptional gene regulation by the hypoxiainducible factor HIF- $1 \alpha$. HIF- $1 \alpha$ protein undergoes rapid prolyl hydroxylation under normoxic conditions by specific cellular prolyl hydroxylases. Direct hydroxylation by ROS is a purported alternative pathway. Hydroxylated HIF interacts with the $\mathrm{VHL}$, a critical member of an $\mathrm{E} 3$ ubiquitin ligase complex that polyubiquitylates HIF (Ub, ubiquitin). Polyubiquitylation targets HIF- $1 \alpha$ for destruction by the proteosome. Under hypoxia $\left(\downarrow \mathrm{O}_{2}\right)$ hydroxylation does not occur and HIF$1 \alpha$ is stabilized. Heterodimerization with ARNT forms the active HIF complex that binds to a core hypoxia response element in a wide array of genes involved in a diversity of biological processes germane to cardiovascular function. Transcriptional activation of iNOS expression is shown as an example of how HIF-mediated gene expression can affect $\mathrm{ROS}$ generation by generating $\mathrm{NO}$ that interacts with $\mathrm{O}_{2}{ }^{--}$to form ONOO--. NOX2 is shown as a cellular source of $\mathrm{O}_{2}{ }^{--}$. dant, prolyl-hydroxylation does not occur, and consequently VHL does not bind to and ubiquitylate HIF- $1 \alpha$. HIF- $1 \alpha$ thus accumulates in the cell, associates with ARNT, binds the HRE in hypoxiaresponsive genes, and alters the transcription of those genes. This post-translational level of control assures that HIF-mediated transcriptional responses to hypoxia occur rapidly. Control of HIF- $1 \alpha$ expression at the mRNA level does occur but is much less important than this post-translational mechanism.

Cardiomyocyte-specific deletion of HIF-1 $\alpha$ leads to altered expression of multiple genes in the normoxic heart, which establishes that HIF-1 $\alpha$ plays an important role in coordinating gene expression with oxygen availability within the normal range of oxygen tensions encountered in the heart, independent of hypoxia or ischemia. This is borne out by the finding that expression of HIF- $1 \alpha$ in cardiomyocytes is required to maintain normal myocardial metabolism, vascularity, calcium handling, and contractile function under normoxic conditions (3). Interestingly, it was recently shown that HIF-1 $\alpha$ levels are also coordinated by a Fenton reaction in the endoplasmic reticulum, which provides an important link between ROS and oxygen-sensitive gene expression (116). Conversely, HIF- $1 \alpha$ coordinates the expression of several genes that could have a significant impact on ROS and nitroso-redox balance $(117,118)$. For example, HIF regulates the expression of iNOS and, indirectly (via VEGF), the levels and activity of eNOS and thus has an effect on NO availability. HIF also has marked effects on the expression of genes involved in cardiac metabolism, including all of the glycolytic enzymes, a critical glucose transporter, and lactate dehydrogenase (3). These alterations in cardiac metabolism could have a significant impact on ROS generation. Evidence that hypoxia-inducible gene expression may play a role in control of oxidative stress includes the demonstration in a recent publication that deletion of HIF-2 $\alpha$ (EPAS-1, an HIF family member that also dimerizes with ARNT) causes multiple organ pathology, including severe cardiomyopathy, associated with loss of ROS homeostasis (46). Figure 3 summarizes the mechanisms by which HIF levels are regulated by oxygen and the manner in which ROS and HIF may interact.
Hypoxia-inducible gene expression via HIF-1 $\alpha$ is beneficially adaptive in the context of acute or subacute myocardial hypoxia or ischemia (119). Examples of HIF-mediated adaptations include increased expression of VEGF to promote angiogenesis; glucose transporter 1 to enhance glucose uptake; the glycolysis-associated enzymes to facilitate glucose metabolism; and erythropoietin to enhance hematopoiesis and increase oxygen carrying capacity. It remains an open question, however, whether chronic activation of HIF- $1 \alpha$, or other hypoxia-responsive pathways, is maladaptive.

Chronic ischemia can lead to progressive heart failure, independent of the acute loss of heart muscle by necrosis. Whether progressive loss of function in the setting of chronic ischemia is due to incremental loss of myocytes and decreased contractility resulting from an inadequate adaptive response to ischemia or is due to maladaptive effects of chronically activated hypoxia-ischemia response pathways in the heart, or both, remains unclear. HIF- $1 \alpha$ levels have been shown to be elevated in the myocardium of patients with ischemic cardiomyopathy (120), and it is conceivable that chronic transcriptional activation of HIF- $1 \alpha$-responsive genes is deleterious. In addition to HIF- $1 \alpha$, there are at least 2 additional HIF subunits, HIF- $2 \alpha$ (EPAS-1) and HIF- $3 \alpha$, that are regulated in a manner similar to HIF- $1 \alpha$ and that can partner with ARNT. The relative roles of HIF- $1 \alpha$ and HIF- $2 \alpha$ remain unclear, and the coordination of their activities appears to be complex (121). As mentioned above, HIF- $2 \alpha$-null mice manifest a severe cardiomyopathy that appears to be related to ROS activation (46). Whether this phenotype is exclusively due to the loss of HIF- $2 \alpha$-mediated effects on gene expression or involves in some reciprocal manner alterations in HIF- $1 \alpha$-mediated activity is not clear from the literature. Interestingly, we have recently found that cardiac-specific deletion of VHL, an intervention that causes an increase in HIF-1 $\alpha$ levels, causes severe dilated cardiomyopathy with pathologic changes consistent with those seen in ischemic cardiomyopathy (122). Whether this is due entirely to elevated HIF-1 $\alpha$ levels in the absence of VHL or to loss of an HIF-independent role of VHL remains unclear, but the data from 
these studies strongly supports a critical role of the HIF pathway in regulating cardiac function.

\section{Is there relationship between ventricular geometry, oxygen, ROS, and cardiac function?}

Ventricular geometry is a major determinant of cardiac function and is also a critical determinant of myocardial oxygen consumption. Myocardial oxygen consumption is proportional to ventricular wall tension, and by Laplace's law, ventricular wall tension is proportional to $P \times r / 2 \pi$ (where $P$ is pressure, $r$ is the radius of curvature of the ventricle, and $\pi$ is ventricular wall thickness). Thus, at any given pressure and myocardial thickness, a larger ventricle will consume more oxygen per gram tissue than a smaller one. Therefore, irrespective of the etiology, a failing dilated heart requires more oxygen per gram tissue than a nonfailing smaller heart. The consequences of this remain unclear, especially in the setting of heart failure unrelated to coronary disease, but it is reasonable to postulate that this alteration in myocardial oxygen consumption leads to alterations in gene expression and possibly in ROS generation. Ventricular volume reduction by aneurysmectomy or partial left ventriculectomy can lead to improved cardiac function, although in the latter case there is no clear correlation with increased survival (123). The role of decreased wall stress, decreased myocardial oxygen consumption, and consequent alterations in the expression of hypoxia-responsive genes in the clinical response to these procedures is unclear but of significant interest. The Acorn device, a mesh that is wrapped around the heart to limit ventricular dilation, is under clinical investigation for the treatment of heart failure. Interestingly, it is postulated that one potential benefit of this approach will be to decrease ROS generation associated with cardiac dilation (124).

One additional, as-yet-unproven therapeutic approach to heart failure is the induction of angiogenesis with growth factors such as VEGF, even in the absence of coronary artery disease. This approach is based on the hypothesis that myocardial hypoxia occurs as a consequence of mismatch in the relationship between myocardial mass, myocardial oxygen demand, myocardial vascularity, and oxygen delivery and that this mismatch contributes to the genesis of heart failure. This can theoretically occur in a variety of settings, including in nonischemic dilated cardiomyopathy as a consequence of the Laplace relationship as describe above or in pathologic cardiac hypertrophy in which myocardial vascularity might not be sufficient for the increased myocardial muscle mass. Supporting this hypothesis is our finding that cardiomyocyte-specific deletion of VEGF in the mouse heart leads to hypovascularity and dilated cardiomyopathy, although this phenotype may also reflect defects in normal development of the myocardium in the absence of VEGF (125).

\section{Conclusions}

Oxygen, beyond its indispensable role in cardiac energy metabolism, plays a central role in other biological processes that can be determinants of cardiac function, including the generation of ROS and the determination of cardiac gene expression patterns. Although their role in the pathogenesis of clinical heart failure remains unclear, ROS have been implicated in most processes thought to have a significant effect on cardiac function, including hypertrophy, ion flux and calcium handling, EC coupling, extracellular matrix configuration, vasomotor function, metabolism, gene expression, and downstream signaling of several growth factors and cytokines. Clinical trials based on antioxidant therapies have been, however, generally disappointing. Whether this is a function of the particular antioxidants used is unclear, and planned trials with XO inhibitors and other alternative agents should help answer this question. The role of hypoxia-induced alterations in gene expression in the genesis of heart failure also remains unclear, although experimental data suggest that these changes in gene expression can be either adaptive or maladaptive, depending on context. Given the central role of oxygen in cardiovascular biology, further investigational focus on oxygen-related processes in the genesis of heart failure is warranted.

Address correspondence to: Frank J. Giordano, Department of Medicine, Yale University School of Medicine, BCMM 436C, 295 Congress Avenue, New Haven, Connecticut 06510, USA. Phone: (203) 785-7361; Fax: (203) 737-2290; E-mail: Frank.Giordano@yale.edu.
1. West, J.B. 1991. Cardiac energetics and myocardial oxygen consumption. Physiologic basis of medical practice. Williams and Wilkins. Baltimore, Maryland, USA. 250-260.

2. Braunwald, E. 2001. Coronary blood flow and myocardial ischemia. Heart disease: a textbook of cardiovascular medicine. W.B. Saunders Company. Philadelphia, Pennsylvania, USA. 1161-1183.

3. Huang, Y., et al. 2004. Cardiac myocyte-specific HIF-1alpha deletion alters vascularization, energy availability, calcium flux, and contractility in the normoxic heart. FASEB J. 18:1138-1140.

4. Davies, K.J. 1995. Oxidative stress: the paradox of aerobic life. Biochem. Soc. Symp. 61:1-31.

5. Jafri, M.S., Dudycha, S.J., and O'Rourke, B. 2001. Cardiac energy metabolism: models of cellular respiration. Annu. Rev. Biomed. Eng. 3:57-81.

6. Ide, T., et al. 1999. Mitochondrial electron transport complex I is a potential source of oxygen free radicals in the failing myocardium. Circ. Res. 85:357-363.

7. Miwa, S., and Brand, M.D. 2003. Mitochondrial matrix reactive oxygen species production is very sensitive to mild uncoupling. Biochem. Soc. Trans. 31:1300-1301.

8. Genova, M.L., et al. 2003. Mitochondrial production of oxygen radical species and the role of Coen- zyme Q as an antioxidant. Exp. Biol. Med. (Maywood). 228:506-513.

9. Toufektsian, M.C., Boucher, F.R., Tanguy, S., Morel, S., and de Leiris, J.G. 2001. Cardiac toxicity of singlet oxygen: implication in reperfusion injury. Antioxid. Redox. Signal. 3:63-69.

10. Seshiah, P.N., et al. 2002. Angiotensin II stimulation of $\mathrm{NAD}(\mathrm{P}) \mathrm{H}$ oxidase activity: upstream mediators. Circ. Res. 91:406-413.

11. Xia, Y., Tsai, A.L., Berka, V., and Zweier, J.L. 1998 Superoxide generation from endothelial nitricoxide synthase. A Ca2+/calmodulin-dependent and tetrahydrobiopterin regulatory process. J. Biol. Chem. 273:25804-25808.

12. Xia, Y., Roman, L.J., Masters, B.S., and Zweier, J.L. 1998. Inducible nitric-oxide synthase generates superoxide from the reductase domain. J. Biol. Chem. 273:22635-22639.

13. Griendling, K.K., Sorescu, D., and Ushio-Fukai, M. 2000. NAD(P)H oxidase: role in cardiovascular biology and disease. Circ. Res. 86:494-501.

14. Sawyer, D.B., et al. 2002. Role of oxidative stress in myocardial hypertrophy and failure. J. Mol. Cell. Cardiol. 34:379-388.

15. Thannickal, V.J., and Fanburg, B.L. 2000. Reactive oxygen species in cell signaling. Am. J. Physiol. Lung Cell Mol. Physiol. 279:L1005-L1028.
16. Bendall, J.K., Cave, A.C., Heymes, C., Gall, N., and Shah, A.M. 2002. Pivotal role of a gp91(phox)containing NADPH oxidase in angiotensin IIinduced cardiac hypertrophy in mice. Circulation. 105:293-296.

17. Wu, M.L., Chan, C.C., and Su, M.J. 2000. Possible mechanism(s) of arachidonic acid-induced intracellular acidosis in rat cardiac myocytes. Circ. Res. 86:E55-E62.

18. Sauer, H., et al. 2004. Involvement of reactive oxygen species in cardiotrophin-1-induced proliferation of cardiomyocytes differentiated from murine embryonic stem cells. Exp. Cell Res. 294:313-324.

19. Heymes, C., et al. 2003. Increased myocardial NADPH oxidase activity in human heart failure. J. Am. Coll. Cardiol. 41:2164-2171.

20. Sabri, A., Hughie, H.H., and Lucchesi, P.A. 2003. Regulation of hypertrophic and apoptotic signaling pathways by reactive oxygen species in cardiac myocytes. Antioxid. Redox Signal. 5:731-740.

21. Nordberg, J., and Arner, E.S. 2001. Reactive oxygen species, antioxidants, and the mammalian thioredoxin system. Free Radic. Biol. Med. 31:1287-1312.

22. Kirkman, H.N., and Gaetani, G.F. 1984. Catalase: a tetrameric enzyme with four tightly bound molecules of NADPH. Proc. Natl. Acad. Sci. U. S. A. 
81:4343-4347.

23. Kirkman, H.N., Rolfo, M., Ferraris, A.M., and Gaetani, G.F. 1999. Mechanisms of protection of catalase by NADPH. Kinetics and stoichiometry. J. Biol. Chem. 274:13908-13914.

24. Ursini, F., et al. 1995. Diversity of glutathione peroxidases. Methods Enzymol. 252:38-53.

25. de Haan, J.B., et al. 2004. Fibroblasts derived from Gpx1 knockout mice display senescent-like features and are susceptible to $\mathrm{H} 2 \mathrm{O} 2$-mediated cell death. Free. Radic. Biol. Med. 36:53-64.

26. Conrad, M., et al. 2004. Essential role for mitochondrial thioredoxin reductase in hematopoiesis, heart development, and heart function. Mol. Cell. Biol. 24:9414-9423.

27. Hensley, K., Robinson, K.A., Gabbita, S.P., Salsman, S., and Floyd, R.A. 2000. Reactive oxygen species, cell signaling, and cell injury. Free Radic. Biol. Med. 28:1456-1462.

28. Nishida, M., et al. 2000. G alpha(i) and G alpha(o) are target proteins of reactive oxygen species. Nature. 408:492-495.

29. Griendling, K.K., and FitzGerald, G.A. 2003. Oxidative stress and cardiovascular injury: part I: basic mechanisms and in vivo monitoring of ROS. Circulation. 108:1912-1916.

30. Machida, Y., et al. 2003. Overexpression of tumor necrosis factor-alpha increases production of hydroxyl radical in murine myocardium. Am. J. Physiol. Heart Circ. Physiol. 284:H449-H455.

31. Hirotani, S., et al. 2002. Involvement of nuclear factor-kappaB and apoptosis signal-regulating kinase 1 in G-protein-coupled receptor agonistinduced cardiomyocyte hypertrophy. Circulation. 105:509-515.

32. Hsu, T.C., Young, M.R., Cmarik, J., and Colburn, N.H. 2000. Activator protein 1 (AP-1)- and nuclear factor kappaB (NF-kappaB)-dependent transcriptional events in carcinogenesis. Free Radic. Biol. Med. 28:1338-1348.

33. Turpaev, K.T. 2002. Reactive oxygen species and regulation of gene expression. Biochemistry Mosc. 67:281-292.

34. Wu, H.M., Chi, K.H., and Lin, W.W. 2002. Proteasome inhibitors stimulate activator protein-1 pathway via reactive oxygen species production. FEBS Lett. 526:101-105.

35. Hemnani, T., and Parihar, M.S. 1998. Reactive oxygen species and oxidative DNA damage. Indian J. Physiol. Pharmacol. 42:440-452.

36. Suematsu, N., et al. 2003. Oxidative stress mediates tumor necrosis factor-alpha-induced mitochondrial DNA damage and dysfunction in cardiac myocytes. Circulation. 107:1418-1423.

37. Rathore, N., John, S., Kale, M., and Bhatnagar, D. 1998. Lipid peroxidation and antioxidant enzymes in isoproterenol induced oxidative stress in rat tissues. Pharmacol. Res. 38:297-303.

38. Thollon, C., Iliou, J.P., Cambarrat, C., Robin, F., and Vilaine, J.P. 1995. Nature of the cardiomyocyte injury induced by lipid hydroperoxides. Cardiovasc. Res. 30:648-655.

39. Rahman, I. 2003. Oxidative stress, chromatin remodeling and gene transcription in inflammation and chronic lung diseases. J. Biochem. Mol. Biol. 36:95-109.

40. Konat, G.W. 2003. H2O2-induced higher order chromatin degradation: a novel mechanism of oxidative genotoxicity. J. Biosci. 28:57-60.

41. Lockwood, T.D. 2000. Redox control of protein degradation. Antioxid. Redox Signal. 2:851-878.

42. Stadtman, E.R., and Levine, R.L. 2003. Free radicalmediated oxidation of free amino acids and amino acid residues in proteins. Amino Acids. 25:207-218.

43. Sinclair, D.A. 2002. Paradigms and pitfalls of yeast longevity research. Mech. Ageing Dev. 123:857-867.

44. Lakatta, E.G. 2003. Arterial and cardiac aging: major shareholders in cardiovascular disease enterprises: part III: cellular and molecular clues to heart and arterial aging. Circulation 107:490-497.

45. Wallace, D.C. 2001. Mouse models for mitochondrial disease. Am. J. Med. Genet. 106:71-93.

46. Scortegagna, M., et al. 2003. Multiple organ pathology, metabolic abnormalities and impaired homeostasis of reactive oxygen species in Epas1-/- mice. Nat. Genet. 35:331-340.

47. Cesselli, D., et al. 2001. Oxidative stress-mediated cardiac cell death is a major determinant of ventricular dysfunction and failure in dog dilated cardiomyopathy. Circ. Res. 89:279-286.

48. Ho, Y.S., Magnenat, J.L., Gargano, M., and Cao, J. 1998. The nature of antioxidant defense mechanisms: a lesson from transgenic studies. Environ. Health Perspect. 106(Suppl. 5):1219-1228.

49. Yen, H.C., Oberley, T.D., Vichitbandha, S., Ho, Y.S., and St. Clair, D.K. 1996. The protective role of manganese superoxide dismutase against adriamycin-induced acute cardiac toxicity in transgenic mice. J. Clin. Invest. 98:1253-1260.

50. Chen, E.P., Bittner, H.B., Davis, R.D., Folz, R.J., and Van Trigt, P. 1996. Extracellular superoxide dismutase transgene overexpression preserves postischemic myocardial function in isolated murine hearts. Circulation. 94:II412-II417.

51. Pennathur, S., Wagner, J.D., Leeuwenburgh, C., Litwak, K.N., and Heinecke, J.W. 2001. A hydroxyl radical-like species oxidizes cynomolgus monkey artery wall proteins in early diabetic vascular disease. J. Clin. Invest. 107:853-860.

52. Khatri, J.J., et al. 2004. Vascular oxidant stress enhances progression and angiogenesis of experimental atheroma. Circulation. 109:520-525.

53. Witztum, J.L., and Steinberg, D. 1991. Role of oxidized low density lipoprotein in atherogenesis. J. Clin. Invest. 88:1785-1792.

54. Rajagopalan, S., Meng, X.P., Ramasamy, S., Harrison, D.G., and Galis, Z.S. 1996. Reactive oxygen species produced by macrophage-derived foam cells regulate the activity of vascular matrix metalloproteinases in vitro. Implications for atherosclerotic plaque stability. J. Clin. Invest. 98:2572-2579.

55. Asimakis, G.K., Lick, S., and Patterson, C. 2002. Postischemic recovery of contractile function is impaired in SOD2(+/-) but not SOD1(+/-) mouse hearts. Circulation. 105:981-986.

56. Yoshida, T., Maulik, N., Engelman, R.M., Ho, Y.S. and Das, D.K. 2000. Targeted disruption of the mouse Sod I gene makes the hearts vulnerable to ischemic reperfusion injury. Circ. Res. 86:264-269.

57. Bolli, R. 1998. Causative role of oxyradicals in myocardial stunning: a proven hypothesis. A brief review of the evidence demonstrating a major role of reactive oxygen species in several forms of postischemic dysfunction. Basic Res. Cardiol. 93:156-162.

58. Perez, N.G., Gao, W.D., and Marban, E. 1998. Novel myofilament Ca2+-sensitizing property of xanthine oxidase inhibitors. Circ. Res. 83:423-430.

59. Charlat, M.I., et al. 1987. Evidence for a pathogenetic role of xanthine oxidase in the "stunned" myocardium. Am. J. Physiol. 252:H566-H577.

60. Pfeffer, M.A., and Braunwald, E. 1990. Ventricular remodeling after myocardial infarction. Experimental observations and clinical implications. Circulation. 81:1161-1172.

61. Mann, D.L., and Spinale, F.G. 1998. Activation of matrix metalloproteinases in the failing human heart: breaking the tie that binds. Circulation. 98:1699-1702.

62. von Harsdorf, R., Li, P.F., and Dietz, R. 1999. Signaling pathways in reactive oxygen species-induced cardiomyocyte apoptosis. Circulation. 99:2934-2941.

63. Engberding, N., et al. 2004. Allopurinol attenuates left ventricular remodeling and dysfunction after experimental myocardial infarction: a new action for an old drug? Circulation. 110:2175-2179.

64. Kwon, S.H., Pimentel, D.R., Remondino, A., Sawyer, D.B., and Colucci, W.S. 2003. $\mathrm{H}(2) \mathrm{O}(2)$ regu- lates cardiac myocyte phenotype via concentrationdependent activation of distinct kinase pathways. J. Mol. Cell. Cardiol. 35:615-621.

65. Li, J.M., Gall, N.P., Grieve, D.J., Chen, M., and Shah, A.M. 2002. Activation of NADPH oxidase during progression of cardiac hypertrophy to failure. Hypertension. 40:477-484.

66. Date, M.O., et al. 2002. The antioxidant N-2-mercaptopropionyl glycine attenuates left ventricular hypertrophy in in vivo murine pressure-overload model. J. Am. Coll. Cardiol. 39:907-912.

67. Higuchi, Y., et al. 2002. Involvement of reactive oxygen species-mediated NF-kappa B activation in TNF-alpha-induced cardiomyocyte hypertrophy. J. Mol. Cell. Cardiol. 34:233-240.

68. Pimentel, D.R., et al. 2001. Reactive oxygen species mediate amplitude-dependent hypertrophic and apoptotic responses to mechanical stretch in cardiac myocytes. Circ. Res. 89:453-460.

69. Ghosh, M.C., Wang, X., Li, S., and Klee, C. 2003. Regulation of calcineurin by oxidative stress. Methods Enzymol. 366:289-304

70. Gopalakrishna, R., Gundimeda, U., and Chen, Z.H. 1997. Cancer-preventive selenocompounds induce a specific redox modification of cysteine-rich regions in $\mathrm{Ca}(2+)$-dependent isoenzymes of protein kinase C. Arch. Biochem. Biophys. 348:25-36.

71. Nakamura, K., et al. 1998. Inhibitory effects of antioxidants on neonatal rat cardiac myocyte hypertrophy induced by tumor necrosis factor-alpha and angiotensin II. Circulation. 98:794-799.

72. Delbosc, S., Cristol, J.P., Descomps, B., Mimran, A., and Jover, B. 2002. Simvastatin prevents angiotensin II-induced cardiac alteration and oxidative stress. Hypertension. 40:142-147.

73. Sugden, P.H., and Clerk, A. 1998. "Stress-responsive" mitogen-activated protein kinases (c-Jun $\mathrm{N}$-terminal kinases and p38 mitogen-activated protein kinases) in the myocardium. Circ. Res. 83:345-352.

74. Takemoto, M., et al. 2001. Statins as antioxidant therapy for preventing cardiac myocyte hypertrophy. J. Clin. Invest. 108:1429-1437. doi:10.1172/ JCI200113350.

75. Izumiya, Y., et al. 2003. Apoptosis signal-regulating kinase 1 plays a pivotal role in angiotensin IIinduced cardiac hypertrophy and remodeling. Circ. Res. 93:874-883.

76. Gotoh, Y., and Cooper, J.A. 1998. Reactive oxygen species- and dimerization-induced activation of apoptosis signal-regulating kinase 1 in tumor necrosis factor-alpha signal transduction. J. Biol. Chem. 273:17477-17482.

77. Yamaguchi, O., et al. 2003. Targeted deletion of apoptosis signal-regulating kinase 1 attenuates left ventricular remodeling. Proc. Natl. Acad. Sci. U. S. A. 100:15883-15888.

78. Rayment, N.B., et al. 1999. Myocyte loss in chronic heart failure. J. Pathol. 188:213-219.

79. Adeghate, E. 2004. Molecular and cellular basis of the aetiology and management of diabetic cardiomyopathy: a short review. Mol. Cell. Biochem. 261:187-191.

80. Remondino, A., et al. 2003. Beta-adrenergic receptor-stimulated apoptosis in cardiac myocytes is mediated by reactive oxygen species/c-Jun NH2terminal kinase-dependent activation of the mitochondrial pathway. Circ. Res. 92:136-138.

81. Kourie, J.I. 1998. Interaction of reactive oxygen species with ion transport mechanisms. Am. J. Physiol. 275:C1-C24.

82. Guerra, L., Cerbai, E., Gessi, S., Borea, P.A., and Mugelli, A. 1996. The effect of oxygen free radicals on calcium current and dihydropyridine binding sites in guinea-pig ventricular myocytes. $\mathrm{Br}$. J. Pharmacol. 118:1278-1284.

83. Kaplan, P., Babusikova, E., Lehotsky, J., and Dobrota, D. 2003. Free radical-induced protein 
modification and inhibition of Ca2+-ATPase of cardiac sarcoplasmic reticulum. Mol. Cell. Biochem. 248:41-47.

84. Nakaya, H., Takeda, Y., Tohse, N., and Kanno, M. 1992. Mechanism of the membrane depolarization induced by oxidative stress in guinea-pig ventricular cells. J. Mol. Cell. Cardiol. 24:523-534.

85. Goldhaber, J.I. 1996. Free radicals enhance $\mathrm{Na}^{+} /$ $\mathrm{Ca} 2+$ exchange in ventricular myocytes. Am.J. Physiol. 271:H823-H833.

86. He, X., et al. 2003. ASK1 associates with troponin $\mathrm{T}$ and induces troponin $\mathrm{T}$ phosphorylation and contractile dysfunction in cardiomyocytes. Am. J. Pathol. 163:243-251.

87. Unger, R.H. 2002. Lipotoxic diseases. Annu. Rev. Med. 53:319-336.

88. Sharma, S., et al. 2004. Intramyocardial lipid accumulation in the failing human heart resembles the lipotoxic rat heart. FASEB J. 18:1692-1700.

89. Finck, B.N., et al. 2003. A critical role for PPARalphamediated lipotoxicity in the pathogenesis of diabetic cardiomyopathy: modulation by dietary fat content. Proc. Natl. Acad. Sci. U. S. A. 100:1226-1231.

90. Balligand, J.L., Kelly, R.A., Marsden, P.A., Smith, T.W., and Michel, T. 1993. Control of cardiac muscle cell function by an endogenous nitric oxide signaling system. Proc. Natl. Acad. Sci. U. S. A. 90:347-351.

91. Bonaventura, J., and Gow, A. 2004. NO and superoxide: opposite ends of the seesaw in cardiac contractility. Proc. Natl. Acad. Sci. U. S. A. 101:16403-16404.

92. Khan, S.A., et al. 2004. Neuronal nitric oxide synthase negatively regulates xanthine oxidoreductase inhibition of cardiac excitation-contraction coupling. Proc. Natl. Acad. Sci. U. S. A. 101:15944-15948.

93. Gaston, B.M., Carver, J., Doctor, A., and Palmer L.A. 2003. S-nitrosylation signaling in cell biology. Mol. Interv. 3:253-263.

94. Paolocci, N., et al. 2000. cGMP-independent inotropic effects of nitric oxide and peroxynitrite donors: potential role for nitrosylation. Am. J. Physiol. Heart Circ. Physiol. 279:H1982-H1988.

95. Hare, J.M. 2004. Nitroso-redox balance in the cardiovascular system. N. Engl. J. Med. 351:2112-2114.

96. Ferdinandy, P., Danial, H., Ambrus, I., Rothery, R.A., and Schulz, R. 2000. Peroxynitrite is a major contributor to cytokine-induced myocardial contractile failure. Circ. Res. 87:241-247.

97. Taylor, A.L., et al. 2004. Combination of isosorbide dinitrate and hydralazine in blacks with heart failure. N. Engl. J. Med. 351:2049-2057.

98. Maack, C., et al. 2003. Oxygen free radical release in human failing myocardium is associated with increased activity of rac1-GTPase and represents a target for statin treatment. Circulation. 108:1567-1574.

99. Mak, S., and Newton, G.E. 2001. The oxidative stress hypothesis of congestive heart failure: radical thoughts. Chest. 120:2035-2046.

100.Jaatinen, P., Saukko, P., and Hervonen, A. 1993. Chronic ethanol exposure increases lipopigment accumulation in human heart. Alcohol Alcohol. 28:559-569.

101.Mak, S., and Newton, G.E. 2004. Redox modulation of the inotropic response to dobutamine is impaired in patients with heart failure. Am. J. Physiol. Heart Circ. Physiol. 286:H789-H795.

102. Cappola, T.P., et al. 2001. Allopurinol improves myocardial efficiency in patients with idiopathic dilated cardiomyopathy. Circulation. 104:2407-2411.

103. Doehner, W., et al. 2002. Effects of xanthine oxidase inhibition with allopurinol on endothelial function and peripheral blood flow in hyperuricemic patients with chronic heart failure: results from 2 placebocontrolled studies. Circulation. 105:2619-2624.

104. Rossig, L., et al. 2001. Vitamin C inhibits endothelial cell apoptosis in congestive heart failure. Circulation. 104:2182-2187.

105.Packer, M., et al. 2001. Effect of carvedilol on survival in severe chronic heart failure. N. Engl. J. Med. 344:1651-1658.

106.Hennekens, C.H., et al. 1996. Lack of effect of longterm supplementation with beta carotene on the incidence of malignant neoplasms and cardiovascular disease. N. Engl. J. Med. 334:1145-1149.

107. Yusuf, S., Dagenais, G., Pogue, J., Bosch, J., and Sleight, P. 2000. Vitamin E supplementation and cardiovascular events in high-risk patients. The Heart Outcomes Prevention Evaluation Study Investigators. N. Engl. J. Med. 342:154-160.

108.Lonn, E. et al. 2002. Effects of vitamin E on cardiovascular and microvascular outcomes in highrisk patients with diabetes: results of the HOPE study and MICRO-HOPE substudy. Diabetes Care. 25:1919-1927.

109. Freudenberger, R.S., et al. 2004. Rationale, design and organisation of an efficacy and safety study of oxypurinol added to standard therapy in patients with NYHA class III - IV congestive heart failure. Expert Opin. Investig. Drugs. 13:1509-1516.

110.Giordano, F.J., and Johnson, R.S. 2001. Angiogenesis: the role of the microenvironment in flipping the switch. Curr. Opin. Genet. Dev. 11:35-40.

111. Ryan, H.E., Lo, J., and Johnson, R.S. 1998. HIF-1 alpha is required for solid tumor formation and embryonic vascularization. EMBO J. 17:3005-3015.

112. Carmeliet, P., et al. 1998. Role of HIF-1alpha in hypoxia-mediated apoptosis, cell proliferation and tumour angiogenesis. Nature. 394:485-490.

113.Iyer, N.V., et al. 1998. Cellular and developmental control of O2 homeostasis by hypoxia-inducible factor 1 alpha. Genes Dev. 12:149-162.

114.Semenza, G.L. 2001. HIF-1, O(2), and the 3 PHDs: how animal cells signal hypoxia to the nucleus. Cell. 107:1-3.

115.Kaelin, W.G., Jr. 2002. Molecular basis of the VHL hereditary cancer syndrome. Nat. Rev. Cancer. 2:673-682.

116.Liu, Q., et al. 2004. A Fenton reaction at the endoplasmic reticulum is involved in the redox control of hypoxia-inducible gene expression. Proc. Natl. Acad. Sci. U. S. A. 101:4302-4307.

117. Cormier-Regard, S., Nguyen, S.V., and Claycomb, W.C. 1998. Adrenomedullin gene expression is developmentally regulated and induced by hypoxia in rat ventricular cardiac myocytes. J. Biol. Chem. 273:17787-17792.

118.Lee, P.J., et al. 1997. Hypoxia-inducible factor-1 mediates transcriptional activation of the heme oxygenase-1 gene in response to hypoxia. J. Biol. Chem. 272:5375-5381.

119.Semenza, G.L. 2004. O2-regulated gene expression: transcriptional control of cardiorespiratory physiology by HIF-1. J. Appl. Physiol. 96:1173-1177; discussion 1170-1172.

120.Lee, S.H., et al. 2000. Early expression of angiogenesis factors in acute myocardial ischemia and infarction. N. Engl. J. Med. 342:626-633.

121.Park, S.K., et al. 2003. Hypoxia-induced gene expression occurs solely through the action of hypoxia-inducible factor 1alpha (HIF-1alpha): role of cytoplasmic trapping of HIF-2alpha. Mol. Cell. Biol. 23:4959-4971.

122.Lei, L., et al. 2004. Malignant transformation of heart muscle in the absence of the von Hippel-Lindau protein. Circulation. 110:III-45.

123. Batista, R.J., et al. 1997. Partial left ventriculectomy to treat end-stage heart disease. Ann. Thorac. Surg. 64:634-638.

124.Gorman, R.C., Jackson, B.M., and Gorman, J.H. 2004. The potential role of ventricular compressive therapy. Surg. Clin. North Am. 84:45-59.

125. Giordano, F.J., et al. 2001. A cardiac myocyte vascular endothelial growth factor paracrine pathway is required to maintain cardiac function. Proc. Natl. Acad. Sci. U. S. A. 98:5780-5785. 